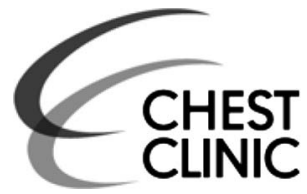

${ }^{1}$ Paediatric Pulmonology and Allergology Department, Hôpitaux pédiatriques de Nice CHU-Lenval, Nice, France ${ }^{2}$ Paediatric Radiology Department, Hôpitaux pédiatriques de Nice $\mathrm{CHU}$ Lenval, Nice, France ${ }^{3}$ Université de Nice-Sophia Antipolis, Nice, France

\section{Correspondence to} Dr Lisa Giovannini-Chami, Paediatric Pneumology and Allergology Department, Hôpitaux pédiatriques de Nice CHU-Lenval, 57 Avenue de la Californie, Nice 06200, France; giovannini-chami.|@pediatriechulenval-nice.fr

Received 27 January 2016 Revised 14 April 2016 Accepted 29 April 2016 Published Online First 23 May 2016
CrossMark

To cite: Fina $A$, BaquéJuston $\mathrm{M}$, Guesmi M, et al. Thorax 2016;71:962-963.

\title{
Symptomatic accessory cardiac bronchus in an infant
}

\author{
A Fina, ${ }^{1}$ M Baqué-Juston, ${ }^{2}$ M Guesmi, ${ }^{2}$ M Albertini, ${ }^{1,3}$ L Giovannini-Chami ${ }^{1,3}$
}

A 13-month-old infant was referred for evaluation of a severe, therapy-resistant asthma. He presented with daily wheezing, recurrent acute exacerbations, chronic dry-to-wet cough and failure to thrive. His mother had severe allergic asthma with recent admission to the intensive care unit. Initial chest X-rays were normal and skin prick tests were negative. It was decided to undertake a comprehensive work-up to exclude differential diagnoses. Paediatric radiologists initially interpreted the chest CT scan as normal. Bronchoscopic examination revealed an accessory cardiac bronchus (ACB) originating from the medial

A

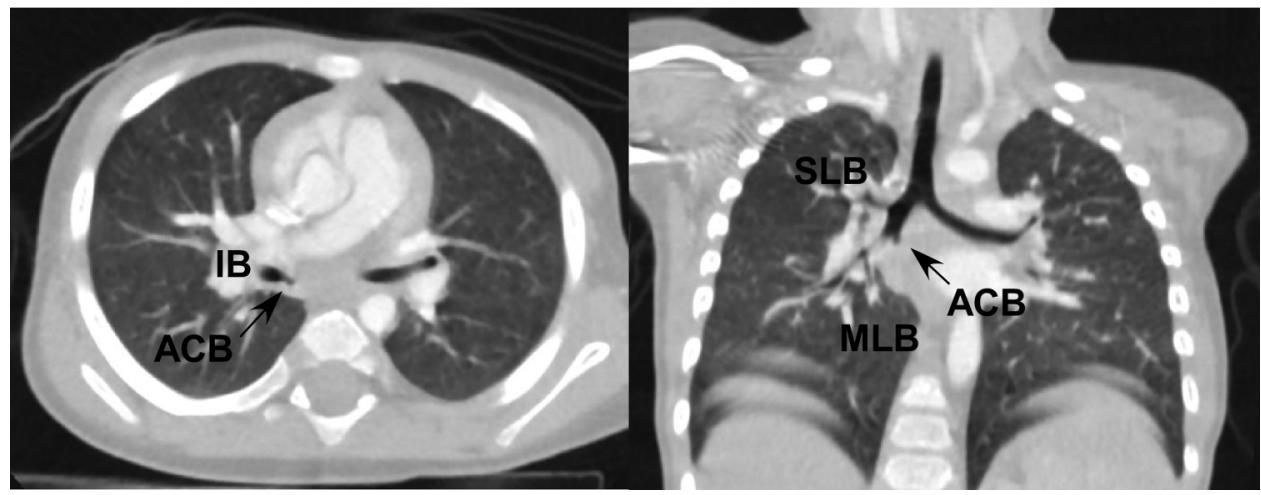

Figure 1 Two-dimensional axial and coronal CT scans. (A) Axial view with the accessory cardiac bronchus (ACB) (arrow) arising from the medial wall of the intermediate bronchus (IB). (B) Coronal reconstructions with vertical and para-cardiac disposition of the ACB (arrow) arising after the superior lobar bronchus (SLB) and above the middle lobar bronchus (MLB), and ending as a blind diverticulum.

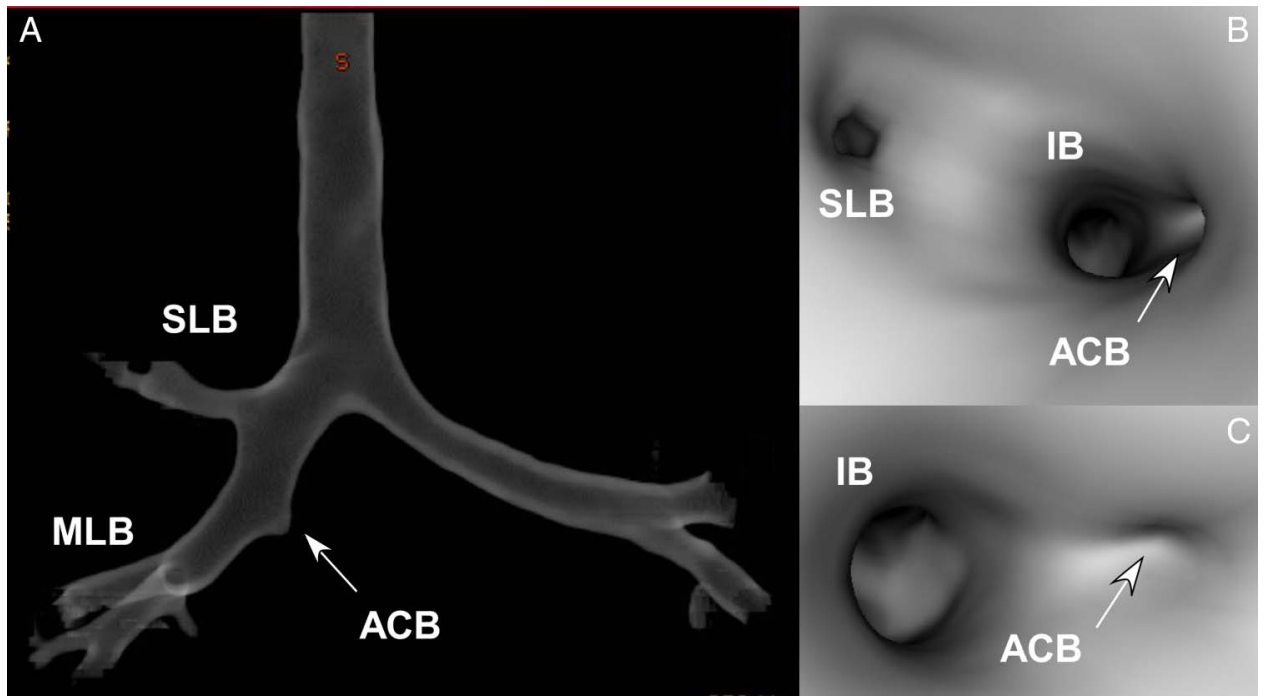

Figure 2 Three-dimensional (3D) surface-rendered reconstructions and virtual bronchoscopy. (A) 3D surface-rendered reconstruction showing the disposition of the accessory cardiac bronchus (ACB) (arrow) on the right bronchial tree with relationship to the remainder of the bronchial tree (superior lobar bronchus (SLB) and middle lobar bronchus $(M L B))$. (B) 3D virtual bronchoscopy showing a view of the entrance of the right main bronchus with the SLB, ACB (arrow) and intermediate bronchus (IB). (C) 3D virtual bronchoscopy showing a view facing the ACB (arrow) with a linear, horizontal collapse just after the orifice of the bronchus. 
abnormal bronchi originating from the trachea or main bronchi remain rare conditions, with tracheal bronchus and ACB being the two most common tracheobronchial variants. ${ }^{1} \mathrm{ACB}$ is an extremely rare condition (incidence $0.09-0.5 \%$ ), usually asymptomatic and discovered incidentally. ${ }^{1}$ ACBs are mostly blind, but can also lead to vestigial or ventilated parenchymal tissue, which must be searched for on CT scan. Blind-ended ACB can act as a reservoir for infected mucus, but forms associated with parenchyma are further exposed to chronic infection, with a major risk of atelectasis and cystic lesions. In adults, symptomatic cases present with haemoptysis, ${ }^{2}$ infections and sometimes malignancies. Although the ACB may not have been the sole cause of his symptoms, the presence of large amounts of infected mucus at the entrance, the collapsed aspect linked to inflammation and leading to impaired drainage, and the localised inflammation on endoscopy support its clinical implication. To our knowledge, this is the first symptomatic ACB described in infancy, or more widely in childhood, the youngest symptomatic subject reported to date being 15 years old. In infants, ACB can initially take the mask of refractory, 'asthma-like' symptoms and can actually be responsible for a chronic, suppurative lung disease.

The diagnosis of ACB may be difficult even in adults and may be delayed. It has been reported that bronchoscopists fail to recognise this abnormality in $22 \%$ of adult cases and radiologists almost always miss it prospectively on two-dimensional (2D) CT scans. ${ }^{3}$ It is nevertheless easily visible on axial and coronal $2 \mathrm{D}$ CT scans, even in an infant, and 3D reconstructions may facilitate the diagnosis.

ACB can be a differential diagnosis of asthma in infancy. Paediatric pulmonologists and radiologists should be aware of this rare condition.

Acknowledgements Thanks to Kate Vassaux, PhD, for manuscript editing. Thanks to Pr Jean-Christophe Dubus for critical revision of the manuscript.

Contributors $A F, M A$ and $L G C$ drafted the manuscript. MG and MBJ provided $C T$ scan reconstructions. LGC made special contributions to critical review of the manuscript. All authors approved the final version of the manuscript.

Competing interests None declared.

Patient consent Obtained.

Provenance and peer review Not commissioned; externally peer reviewed.

\section{REFERENCES}

1 Wooten C, Patel S, Cassidy L, et al. Variations of the tracheobronchial tree: anatomical and clinical significance. Clin Anat 2014;27:1223-33.

2 Keane MP, Meaney JF, Kazerooni EA, et al. Accessory cardiac bronchus presenting with haemoptysis. Thorax 1997:52:490-1.

3 Ghaye B, Kos X, Dondelinger RF. Accessory cardiac bronchus: 3D CT demonstration in nine cases. Eur Radiol 1999:9:45-8. 